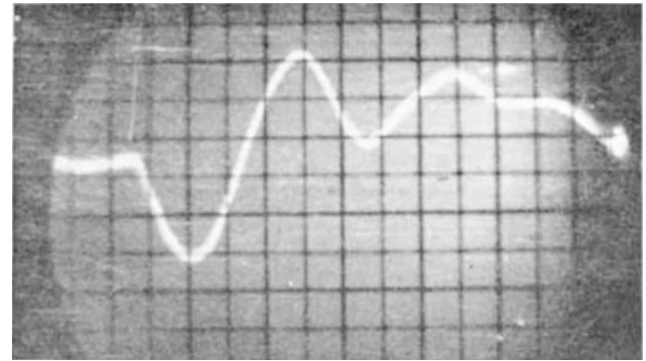
Fig. 3. Rarefaction pulse formed by method $(b)$ measured at

tube near its open end. Even some distance along the propagation tube from the open end, the many wavefronts of these secondary waves are not perpendicular to the tube axis and are still altering their relative orientations. Only at an infinite distance from the open end does the reflected wave system become geometrically stable, and only then is it permissible to compare the experimental results with theory.

So far as is known, the occurrence and behaviour of sound pulses of finite amplitude in which the first half-cycle is one of rarefaction have not previously been recorded in the literature. The reflexion of a compression pulse at any boundary of decreasing acoustic impedance will give rise to a wave of the type described here; for example, an underwater explosion reflected from the free surface of the water. It also seems probable that such a rarefaction pulse might be formed as a result of cavitation phenomena.

G. J. BARBER

T. F. W. EMBleton

Physics Department,

Imperial College of Science and Technology, London, S.W.7. Sept. 7.

1 Timbrell, V., Nature, 167, 306 (1951).

${ }^{2}$ Stokes, G. G., Phil. Mag., (3), 33, 349 (1948)

${ }^{3}$ Chester, W., Phil. Trans. Roy. Soc., A, 242, 527 (1950).

\section{Sequential Sampling in Biological Assay}

Is an investigation relating to the isolation and purification of chorionic gonadotrophin from pregnancy urine, the following problem has arisen : "Does fraction $A$ contain at least the amount $X$ of chorionic gonadotrophin ?" It was necessary to answer this question in the shortest possible time and to use as little material of $A$ as possible. As the solution of this rather specialized problem may have a much more general application to researches of similar nature, it is felt to be worth communicating.

For simplicity, arbitrary concrete figures will be given in the following. Substance $A$ was to be tested for the hypothesis that its chorionic gonadotrophin content was equal to or greater than 100 I.U. per mgm. ; if so, it was to be accepted; if it contained less than 100 I.U./mgm. it was to be discarded. The tolerated risks with regard to the precision of the estimate were fixed at $80-125$ per cent, and with regard to the probability of making a wrong decision were allowed to be 5 per cent, no preference being given to either errors of the first or second kind That is to say, only in five out of two hundred cases would the substance be rejected if it contained 125 or more I.U. chorionic gonadotrophin/mgm., and in only five out of two hundred cases would it be accepted although it actually contained 80 or less I.U./mgm.
In principle, the problem thus defined is a common one in sampling inspection, and a sequential test ${ }^{1}$ seemed most likely to meet the requirements. In the present case the problem is complicated, since no chemical method is suitable and a biological assay method has to be adopted; the spermiation test utilizing male toads ${ }^{2}$ was chosen.

To solve the problem as stated above, substance $A$ was assumed to contain 100 I.U. chorionic gonadotrophin/mgm., and the median effective dose ( $E D 50$ ), according to this assumption, was injected into male toads (Bufo viridis). The toads were taken at random from a population the dose-response curve of which for chorionic gonadotrophin had been established not more than one month before. This seemed justifiable in view of the fact that the slope of the regression line (plotting probit of percentage reactors against log dose) had remained constant within sampling error over a period of two years, and no significant shift of the regression line was ever observed within a period of up to five weeks ${ }^{3}$. The percentage of expected non-reactors (that is, toads with no spermatozoa in their urine $3 \mathrm{hr}$. after the injection), when given 80 and 125 per cent respectively of the $E D 50$, was read off the regression line and was found to be 73 and 27 per cent respectively. Thus, in terms of a sequential test-adopting Wald's ${ }^{1}$ notation, $p$ denoting the fraction of non-reactors-the following values were obtained: $p_{0}=0 \cdot 27, p_{1}=0 \cdot 73$, the probability of committing an error of the first $(\alpha)$ or second $(\beta)$ kind being $\alpha=\beta=0.025$. Consequently, the hypothesis, that the toad population subsequent to the injection of what was assumed to be the ED 50 consisted of 50 per cent or more reactors, was tested against the alternative hypothesis that it contained less than 50 per cent reactors. Thus the test showed whether the dose of chorionic gonadotrophin each toad had received was, in fact, equal to or greater than $E D 50$, and consequently, whether the amount contained in substance $\boldsymbol{A}$ conformed to the requirements for its acceptance.

The actual test was carried out according to standard sequential sampling procedures. Acceptance and rejection numbers were calculated, and the test was terminated as soon as the number of non-reactors agreed with either the acceptance number or the rejection number. Furthermore, to arrive at a decision within $24 \mathrm{hr}$., grouping was adopted (four toads being tested at a time), and the test was truncated if no decision was obtained after the eighth sample (that is, after a total of thirty-two toads had been injected and inspected $3 \mathrm{hr}$. afterwards). Calculation of the average sample number function showed that, on the average, a decision was to be expected after one to four samples, depending on the amount of chorionic gonadotrophin actually contained in the test substance. The actual results were consistent with these theoretical expectations.

Several points, for example, the most practical grouping, the effect of truncation on the probability of making a wrong decision, etc., are still under examination. The results of these investigations will be published in more detail elsewhere.

Euisabeth Bukovics

F. X. WOHLZOGEN

Institute of Physiology,

University of Vienna.

$$
\text { Jan. 22, } 1953 .
$$

1 Wald, A., "Sequential Analysis" (John Wiley, Inc., New York, 1947). 2 Frazer, J. F. D., and Wohlzogen, F. X., J. Physiol., 113, 322 (1951). s Wohlzogen, F. X., Arch. exp. Path. Pharmakol. (in the press). 